\title{
Inhibitory Guanosine Triphosphate-binding Protein-mediated Regulation of Vasopressin Action in Isolated Single Medullary Tubules of Mouse Kidney
}

\author{
Kenmei Takaichi and Kiyoshi Kurokawa \\ Fourth Department of Internal Medicine, University of Tokyo School of Medicine, Tokyo 112, Japan
}

\begin{abstract}
Vasopressin (AVP) plays a key role in maximal urine concentration by stimulating $\mathrm{NaCl}$ reabsorption in the medullary thick ascending limbs of Henle (MAL) and by increasing water permeability in the medullary collecting tubules (MCT). These effects of AVP in MAL and MCT are mediated by cAMP. Alpha ${ }_{2}$-adrenergic stimulation in MCT, and high ambient $\mathrm{Ca}^{2+}$ and $\mathrm{PGE}_{2}$ in MAL inhibit AVP-dependent CAMP production and thereby modulate urine concentration. The present study was undertaken to clarify the mechanisms underlying the inhibition of AVP-dependent CAMP production by these agents using microdissected mouse MAL and MCT. Preincubation of MCT and MAL with $1 \mu \mathrm{g} / \mathrm{ml}$ pertussis toxin for 3 and $6 \mathrm{~h}$, respectively, resulted in ADP-ribosylation of an 41-kD protein, which was presumably an alpha subunit of the inhibitory GTP-binding protein $G_{i}$. Epinephrine, $10^{-6} \mathrm{M}$, via alpha $2^{-}$ adrenergic stimulation, inhibited AVP-dependent cAMP production in MCT. Preincubation of MCT for $3 \mathrm{~h}$ with pertussis toxin abolished the inhibition of AVP-dependent cAMP production by epinephrine. High ambient $\mathrm{Ca}^{2+}$ and $\mathrm{PGE}_{2}$ both inhibited AVP-dependent cAMP production in MAL. Preincubation of MAL for $6 \mathrm{~h}$ with pertussis toxin abolished the inhibition by high ambient $\mathrm{Ca}^{2+}$ and attenuated the inhibition by $\mathrm{PGE}_{2}$. Preincubation of MCT or MAL with pertussis toxin for $1 \mathrm{~h}$ was ineffective in ADP-ribosylation and did not modify the inhibition of AVP-dependent cAMP production by these agents in both nephron segments. Our data suggest that the inhibition of AVP-dependent cAMP production by alpha adrenergic stimulation in MCT, and by high ambient $\mathrm{Ca}^{2+}$ and $\mathrm{PGE}_{2}$ in $\mathrm{MAL}$, is mediated, at least in part, through activation of $\mathbf{G}_{\mathbf{i}}$.
\end{abstract}

\section{Introduction}

Vasopressin (AVP) ${ }^{1}$ plays a central role in maximal urine concentration by stimulating $\mathrm{NaCl}$ reabsorption in medullary

A portion of this study was presented at the annual meeting of the American Society of Nephrology, Washington, D.C., December 13-16, 1987, and appeared as an abstract in Kidney Int. 33:287, 1988. Address correspondence to Dr. Kenmei Takaichi, Fourth Department of Internal Medicine, University of Tokyo School of Medicine, 3-28 Mejirodai, Bunkyo-ku, Tokyo 112, Japan.

Received for publication 20 October 1987 and in revised form 4 May 1988.

1. Abbreviations used in this paper: AVP, vasopressin; MAL, medullary thick ascending limbs of Henle; MCT, medullary collecting tubules; MIX, methylisobutylxanthine; $G_{i}$, inhibitory GTP-binding protein; $\mathrm{G}_{\mathbf{s}}$, stimulatory $\mathrm{GTP}$-binding protein.

J. Clin. Invest.

(c) The American Society for Clinical Investigation, Inc.

$0021-9738 / 88 / 10 / 1437 / 08 \$ 2.00$

Volume 82, October 1988, 1437-1444 thick ascending limbs of Henle (MAL), thus generating the medullary hypertonicity by increasing the water permeability of collecting tubules and allowing water absorption from the urine along the osmotic gradient. These effects of AVP in both nephron segments are mediated by activation of adenylate cyclase (1-8). Activation of adenylate cyclase by a hormone involves binding of the hormone to its specific receptor, activation of stimulatory GTP-binding protein $\mathrm{G}_{\mathrm{s}}$, and activation of the catalytic unit of the enzyme. Several hormones or factors are known to inhibit adenylate cyclase activity through activation of inhibitory GTP-binding protein $G_{i}(9)$.

Several agents have been reported to modulate urine concentration by inhibiting AVP-dependent cAMP production in collecting tubules and MAL. Thus, it has been shown that alpha $a_{2}$-adrenergic agonists inhibit AVP-dependent cAMP production and thereby suppress water permeability in collecting tubules, while $\mathrm{PGE}_{2}$ and high ambient $\mathrm{Ca}^{2+}$ inhibit AVP-dependent cAMP production in MAL (10-14). Indeed, $\mathrm{PGE}_{2}$ suppresses AVP-stimulated $\mathrm{NaCl}$ reabsorption in MAL by inhibiting AVP-dependent cAMP production (15).

The mechanisms underlying the inhibition of AVP-dependent cAMP production by these agents have been poorly understood. Nonetheless, according to the data obtained in other tissues $(16,17)$, it is likely that the effects of alpha ${ }_{2}$-adrenergic stimulation in collecting tubules are mediated through the activation of inhibitory GTP-binding protein $G_{i}$ of the adenylate cyclase system. It is possible that the inhibition of AVP-dependent cAMP production by high ambient $\mathrm{Ca}^{2+}$ and $\mathrm{PGE}_{2}$ might also be mediated through $\mathrm{G}_{\mathrm{i}}$. Thus, our present study was undertaken to examine whether these agents inhibit AVPdependent cAMP production in renal tubules through the activation of $G_{i}$. To this end, we used pertussis toxin, an agent known to specifically inhibit $G_{i}$-mediated suppression of adenylate cyclase activity by ADP-ribosylation of the alpha subunit of $G_{i}$ (18). The results of our study suggest that alpha adrenergic stimulation in $\mathrm{MCT}$, and $\mathrm{PGE}_{2}$ and high ambient $\mathrm{Ca}^{2+}$ in MAL suppress AVP-dependent cAMP production through the activation of $G_{i}$.

\section{Methods}

Synthetic AVP (grade VI), collagenase (type 1), (-)-epinephrine, DLpropranolol, and yohimbine were purchased from Sigma Chemical Co. (St. Louis, MO). ${ }^{32}$ P-NAD was purchased from New England Nuclear (Boston, MA). Pertussis toxin (islet-activating protein) was purchased from Kaken Seiyaku (Tokyo).

Isolation of single nephron segments was performed in a fashion similar to that reported previously from our laboratory with minor modifications in the buffer media used $(13,14,19,20)$. In the present study, we used Dulbecco's modified Eagle-minimal essential medium (DMEM) containing 0.1\% BSA, $24 \mathrm{mM} \mathrm{NaHCO}_{3}$, and $25 \mathrm{mM}$ Hepes, pH 7.4, equilibrated with $95 \% \mathrm{O}_{2}$ and $5 \% \mathrm{CO}_{2}$ (DMEM-Hepes medium). Male ICR mice weighing $\sim 30 \mathrm{~g}$ were lightly anesthetized with an intraperitoneal injection of sodium pentobarbital, $2 \mathrm{mg} / \mathrm{mouse}$. The left kidney was perfused with ice-cold DMEM-Hepes medium containing $0.1 \%$ collagenase. The left kidney was removed and slices of 
$\sim 0.5-1.0-\mathrm{mm}$ thickness were incubated at $37^{\circ} \mathrm{C}$ for $10 \mathrm{~min}$ in the same medium under $95 \% \mathrm{O}_{2}$ and $5 \% \mathrm{CO}_{2}$. After collagenase treatment, kidney slices were rinsed extensively with ice-cold dissection medium (see below). MAL and MCT were dissected from the outer medulla under a stereomicroscope in ice-cold $\left(0-5^{\circ} \mathrm{C}\right)$ dissection medium. The composition of the dissection medium was (in millimolars): $\mathrm{NaCl}, 137 ; \mathrm{KCl}, 5 ; \mathrm{Na}_{2} \mathrm{HPO}_{4}, 0.33 ; \mathrm{KH}_{2} \mathrm{PO}_{4}, 0.44 ; \mathrm{MgSO}_{4}, 0.8$; $\mathrm{MgCl}_{2}, 1.0 ; \mathrm{CaCl}_{2}, 0.25$; glucose, 5.5; and Hepes, $10 ; \mathrm{pH} 7.4$, and with $0.025 \%$ BSA.

In vitro ADP-ribosylation was examined in accordance with the methods described by Okajima et al. (21) with slight modifications. Appropriate pieces of MAL and MCT were transferred with $10 \mu \mathrm{l}$ of dissection medium into $100 \mu \mathrm{l}$ DMEM-Hepes buffer with or without 1 $\mu \mathrm{g} / \mathrm{ml}$ pertussis toxin placed on the bottom of siliconized biological slides kept on ice. Tubular length was measured through a drawing tube attached to the microscope as reported previously $(13,14,19,20)$. After preincubation under $95 \% \mathrm{O}_{2}$ and $5 \% \mathrm{CO}_{2}$ at $37^{\circ} \mathrm{C}$ with a moist atmosphere for varied lengths of time as specified in the Results, incubation medium was aspirated and tubules were washed three times with $100 \mu$ l DMEM-Hepes medium without pertussis toxin under stereomicroscopic observation. After aspiration of the final rinse, the medium was changed to $20 \mu \mathrm{l}$ hypotonic solution. The composition of the hypotonic solution was (in millimolars): $\mathrm{MgCl}_{2}, 5$; EGTA, 1; Tris$\mathrm{HCl}, 25 ; \mathrm{pH} 7.5$, with $1 \mathrm{mg} / \mathrm{ml}$ aprotinin. After freezing and thawing repeated three times to permeabilize the tubular cell membrane, the hypotonic medium was changed to $40 \mu \mathrm{l}$ of reaction medium. The composition of the reaction medium was (in millimolars): Tris- $\mathrm{HCl}$, 25; EGTA, 1; $\mathrm{MgCl}_{2}$, 2.5; ATP, 1; DTT, 2; thymidine, 10 ; ${ }^{32} \mathrm{P}-\mathrm{NAD}$, $0.01 ; \mathrm{pH} 7.6$, with $0.1 \mathrm{mg} / \mathrm{ml}$ aprotinin and $25 \mu \mathrm{g} / \mathrm{ml}$ pertussis toxin. Tubules were incubated in the reaction medium at $37^{\circ} \mathrm{C}$ for $20 \mathrm{~min}$, then cooled on ice, and the medium was changed to $50 \mu \mathrm{l}$ of $50 \mathrm{mM}$ Tris- $\mathrm{HCl}$ buffer, $\mathrm{pH} 6.8$, containing $1 \%$ SDS, $2 \% \beta$-mercaptoethanol, and $10 \%$ glycerol. Samples were transferred to test tubes, incubated at $100^{\circ} \mathrm{C}$ for $5 \mathrm{~min}$, and applied to SDS-PAGE. After electrophoresis, an autoradiogram of the gel was obtained after a 10-d exposure time. At each step of a medium change, the medium was aspirated carefully under stereomicroscope so as not to aspirate the tubules; tubules were then washed two to three times with appropriate medium used for the following procedure.

Incubation of tubules with AVP and the assay of CAMP were performed with slight modifications to those reported previously from our laboratory $(13,14,19,20,22)$. Immediately after the transfer of appropriate pieces of MAL and MCT with $2-\mu l$ dissection medium into $20 \mu \mathrm{l}$ DMEM-Hepes medium with or without $1 \mu \mathrm{g} / \mathrm{ml}$ pertussis toxin placed on the bottom of a siliconized test tube, the tube was kept in the ice-cold water bath. After measurement of tubular length, the tubules were preincubated at $37^{\circ} \mathrm{C}$ for several hours under $95 \% \mathrm{O}_{2}$ and $5 \% \mathrm{CO}_{2}$ with a moist atmosphere as specified in the Results. In preliminary studies, we found that evaporation of the medium was $<2 \%$ during this incubation period in our experimental condition. After the preincubation with or without pertussis toxin, $20 \mu \mathrm{l}$ DMEM-Hepes medium containing $2.4 \mathrm{mM}$ methylisobutylxanthine (MIX) (prewarmed to $37^{\circ} \mathrm{C}$ ), AVP, and test agents were added, and the incubation was continued for $3 \mathrm{~min}$. In the experiments of high medium $\mathrm{Ca}^{2+}$ concentration, $\mathrm{Ca}^{2+}$ concentration was raised only after preincubation, i.e., at the same time of the addition of MIX and AVP. Separate aliquots of tubules were incubated in parallel without AVP but with MIX and test agents to determine the basal cAMP production, i.e., the sum of cAMP produced during preincubation and AVP-independent CAMP production during a 3-min incubation with MIX and test agents. Basal cAMP production without AVP in every experimental condition of the present study was always low $(<10 \mathrm{fmol} / \mathrm{mm}$ in $\mathrm{MCT}$ and $<5 \mathrm{fmol} /$ mm in MAL), compared with AVP-dependent cAMP production as shown in the Results. After a 3-min incubation with or without AVP, the reaction was terminated by the addition of $50 \mu \mathrm{l}$ of $10 \%$ TCA. The TCA was extracted three times with $1 \mathrm{ml}$ ether saturated with water and the residual aqueous phase was dried at $37-42^{\circ} \mathrm{C}$. Samples were kept at $-20^{\circ} \mathrm{C}$ until cAMP determination using a RIA assay kit from
Yamasa Shoyu (Tokyo). The alpha-adrenergic stimulation was tested by adding epinephrine together with propranolol. The concentration of AVP used was $10^{-8} \mathrm{M}$, which is near the maximal concentration needed to stimulate cAMP production in MAL and MCT, based on the results of our previous studies $(13,14,19,20)$. In each experiment, the tubules from the same animal were preincubated for the same length of time with or without pertussis toxin as time-controlled experiments.

The determination of the adenylate cyclase activity was performed in a similar fashion to those previously reported from our laboratory with slight modifications (19). The dissected tubules were permeabilized by freezing and thawing two times in a hypotonic solution. The composition of the hypotonic solution was (in millimolars): $\mathrm{Tris}-\mathrm{HCl}$, $8 ; \mathrm{MgCl}_{2}, 1.0$; EGTA, $0.25 ; \mathrm{pH} 7.4$, with $0.1 \%$ BSA. The tubules were transferred with $2 \mu \mathrm{l}$ of hypotonic solution into $8 \mu \mathrm{l}$ of prereaction medium placed on the bottom of the siliconized test tube in the icecold water bath. The composition of the prereaction medium was (in millimolars): Tris- $\mathrm{HCl}, 100 ; \mathrm{MgCl}_{2}, 3.8$; EGTA, 0.25; $\mathrm{MIX}, 1.5 ; \mathrm{pH}$ 7.4, with $0.1 \% \mathrm{BSA}$, and different $\mathrm{Ca}^{2+}$ concentrations. Final concentrations of $\mathrm{Ca}^{2+}$ added are shown in the Results. After measurement of the tubular length, $10 \mu \mathrm{l}$ of reaction medium was added and tubules were incubated for $30 \mathrm{~min}$ at $30^{\circ} \mathrm{C}$. The composition of the reaction medium was (in millimolars): Tris- $\mathrm{HCl}, 100 ; \mathrm{MgCl}_{2}, 3.8$; EGTA, 0.25; MIX, 1.5; ATP, 1.2; phosphocreatine, 40; creatine phosphokinase, 2 $\mathrm{mg} / \mathrm{ml}, \mathrm{pH} 7.4$, with $0.1 \% \mathrm{BSA}$, and with AVP at a final concentration of $10^{-8} \mathrm{M}$. The reaction was terminated by the addition of $20 \mu \mathrm{l}$ of $10 \%$ TCA, and the CAMP generated was determined by RIA after the extraction of TCA with water-saturated ether.

Each experiment consisted of more than triplicate incubations for each experimental condition using nephron segments dissected from one mouse. The mean of the cAMP values obtained in each condition was taken as one value or a single data point. In almost all experiments, the same experiments were repeated using tubules from more than four mice and the data were expressed as the mean \pm SE with the individual mean value from one mouse as a single data point. Data were analyzed by paired or nonpaired $t$ test as appropriate, and the $P$ value $<0.05$ was considered significant.

\section{Results}

The results of in vitro ADP-ribosylation in dissected MCT and MAL are shown in Fig. 1. In MCT, a band at an $\sim 41-\mathrm{kD}$ protein was visible after a 1-h preincubation with or without pertussis toxin, and after a 3-h preincubation without pertussin toxin. This band disappeared specifically after a 3-h preincubation with pertussis toxin. The molecular weight of this band is comparable to that of the alpha subunit of inhibitory GTP binding protein $G_{i}$. In MAL, the band of the same molecular weight protein disappeared after a 6-h preincubation with pertussis toxin, but the band was very visible after a 1-h preincubation with or without pertussis toxin, and after a 6-h preincubation without pertussis toxin. These results indicate that, in our experimental condition, pertussis toxin ADP-ribosylates the alpha subunit of $G_{i}$ in 3 and $6 \mathrm{~h}$ in MCT and MAL, respectively, and that a 1-h preincubation with pertussis toxin is not sufficient for ADP-ribosylation of the alpha subunit of $G_{i}$ in these nephron segments. It should be noted that our present results of ADP-ribosylation and its modulation by pertussis toxin are rather qualitative and may not allow strict quantitative analyses. Because of a minute size of the specimens, autoradiograms were obtained after $10 \mathrm{~d}$ of exposure and the gels were not analyzed by isotopic counting.

Data from our and other laboratories have shown that alpha $_{2}$-adrenergic stimulation inhibits AVP-dependent cAMP production in MCT but not in MAL in rats (12), that $5 \mathrm{mM}$ 
MCT

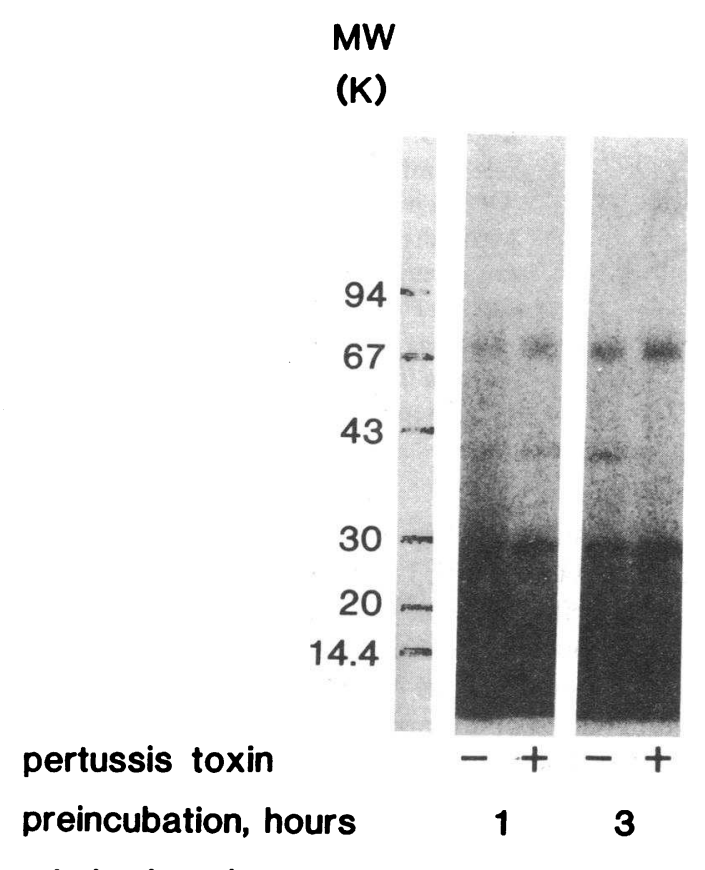

6.74 .76 .16 .3

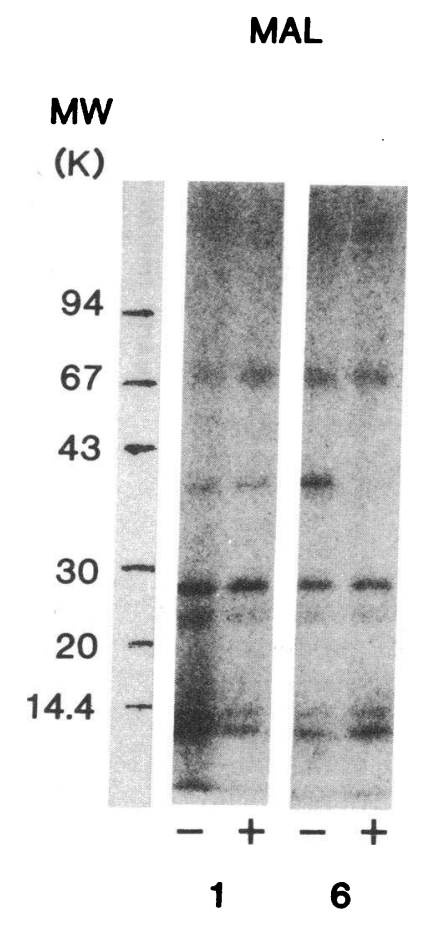

12.918 .216 .912 .5
Figure 1. In vitro ADP-ribosylation in MCT and MAL. After preincubation of dissected MCT and MAL with or without pertussis toxin, tubules were permeabilized by repeated freeze-thawing in hypotonic solution and then incubated with ${ }^{32} \mathrm{P}-\mathrm{NAD}$ and pertussis toxin. After preincubation with pertussis toxin for $3 \mathrm{~h}$ in MCT and for $6 \mathrm{~h}$ in MAL, the band of $\sim 41-\mathrm{kD}$ protein specifically disappeared. Tubular length used in millimeters is depicted on the bottom. See text for details.
$\mathrm{Ca}^{2+}$ inhibits AVP-dependent cAMP production in MAL but not in MCT in mice (14), and that $\mathrm{PGE}_{2}$ inhibits AVP-dependent CAMP production in MAL but not in MCT in rats (13). As shown in Figs. 2 and 3, in the mouse kidney, alpha ${ }_{2}$-adrenergic stimulation inhibited AVP-dependent cAMP production in MCT but only minimally, if at all, in MAL. Both $5 \mathrm{mM}$ $\mathrm{Ca}^{2+}$ and $\mathrm{PGE}_{2}$ inhibited AVP-dependent cAMP production in MAL but not in MCT, results which are similar to those previously reported in rats and mice (12-14). Thus, in subsequent studies, we attempted to clarify the mechanisms underlying the inhibition of AVP-dependent cAMP production by alpha $_{2}$-adrenergic stimulation in MCT and by $5 \mathrm{mM} \mathrm{Ca}^{2+}$ and $\mathrm{PGE}_{2}$ in MAL when both have been dissected from the mouse kidney.

A concentration of $10^{-6} \mathrm{M}$ was chosen for epinephrine, because this concentration showed significant inhibition of
AVP-dependent cAMP production in MCT (Fig. 4). Moreover, this inhibition was recovered by a specific alpha ${ }_{2}$-adrenergic antagonist yohimbine, indicating that this dose of epinephrine suppresses AVP-dependent cAMP production in MCT by activating alpha $a_{2}$-adrenergic receptors.

As shown in Fig. 5, the inhibition of AVP-dependent cAMP production by $10^{-6} \mathrm{M}$ epinephrine was not observed in MCT preincubated with pertussis toxin for $3 \mathrm{~h}$. The pertussis toxin treatment did not affect AVP-dependent cAMP production in the absence of epinephrine. Basal cAMP production in each condition, i.e., the sum of cAMP produced during preincubation and that produced during a 3-min incubation with MIX but without AVP, was always very low, $<10 \mathrm{fmol} / \mathrm{mm}$. The effect of pertussis toxin is most likely due to its specific action of ADP-ribosylating the alpha subunit of $G_{i}$. Thus, after preincubation of MCT with pertussis toxin for $1 \mathrm{~h}$, when the
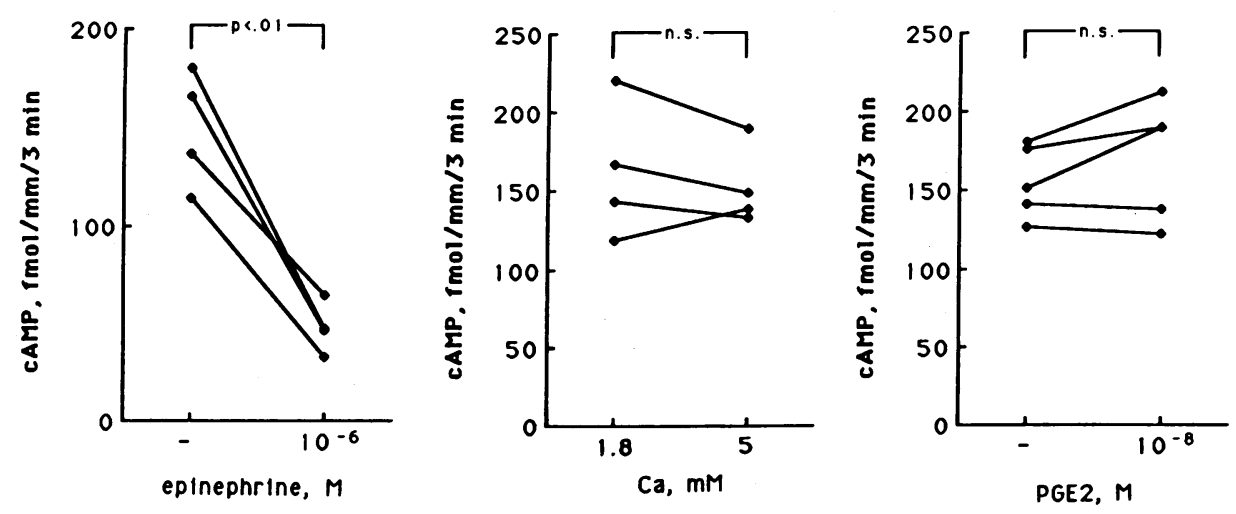

Figure 2. Effects of $10^{-6} \mathrm{M}$ epinephrine, $5 \mathrm{mM} \mathrm{Ca}^{2+}$, and $10^{-8} \mathrm{M} \mathrm{PGE}_{2}$ on AVP-dependent cAMP production in MCT. After preincubation of MCT for 6 min without pertussis toxin, AVP, MIX, and test agents were added and incubated for $3 \mathrm{~min}$. The effect of epinephrine was examined in the presence of $2 \times 10^{-6} \mathrm{M}$ propranolol. Each data point is the mean of values obtained from MCT of one animal and the line connecting data points indicates the results obtained in the tubules of the same animal. Epinephrine, but not high ambient $\mathrm{Ca}^{2+}$ nor $\mathrm{PGE}_{2}$, inhibited AVP-dependent cAMP production in MCT. 


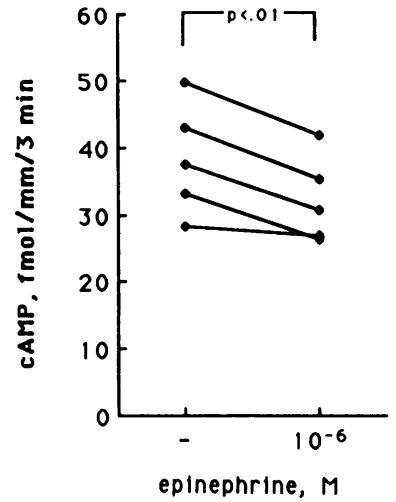

epinephrine, $M$

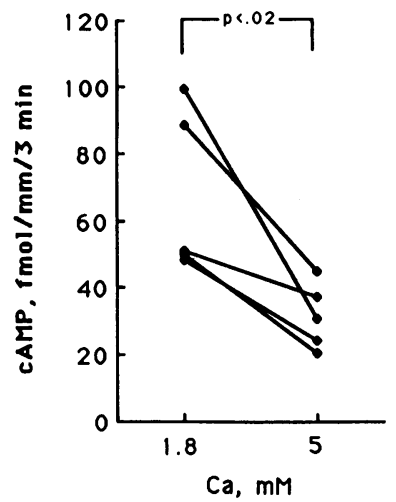

alpha subunit of $G_{i}$ has not yet been ADP-ribosylated (Fig. 1), epinephrine inhibited AVP-dependent cAMP production to a similar degree in MCT preincubated without pertussis toxin, as shown in Fig. 6.

We next examined the involvement of $G_{i}$ in the inhibition by high ambient $\mathrm{Ca}^{2+}$ of AVP-dependent cAMP production in MAL. In this set of experiments, we examined the effects of $5 \mathrm{mM} \mathrm{Ca}^{2+}$, because this concentration of $\mathrm{Ca}^{2+}$ reversibly suppresses AVP-dependent cAMP production in mouse MAL (14).

As shown in Fig. 7, $5 \mathrm{mM} \mathrm{Ca}^{2+}$ in the incubation medium inhibited AVP-dependent cAMP production in MAL after a 6-h preincubation without pertussis toxin. However, the inhibition by high ambient $\mathrm{Ca}^{2+}$ was abolished by prior incubation of MAL with pertussis toxin for $6 \mathrm{~h}$. Basal cAMP production in the absence of AVP with or without pertussis toxin treatment was very low, i.e., $<5 \mathrm{fmol} / \mathrm{mm}$, compared with that in the presence of AVP. The effect of pertussis toxin in MAL is not due to its nonspecific action other than its specific ADPribosylating action of $\mathrm{G}_{i}$, because high $\mathrm{Ca}^{2+}$ in the incubation medium suppressed AVP-dependent cAMP production to a similar degree after preincubation with or without pertussis toxin for $1 \mathrm{~h}$ as shown in Fig. 8. Interestingly, AVP-dependent cAMP production in $1.8 \mathrm{mM} \mathrm{Ca}^{2+}$ (control condition) became greater with pertussis toxin treatment for $6 \mathrm{~h}$, but not for $1 \mathrm{~h}$, than those without pertussis toxin treatment (Figs. 7 and 8).

Our previous data indicate that high ambient $\mathrm{Ca}^{2+}$, per se, may inhibit AVP-dependent cAMP production in MAL but

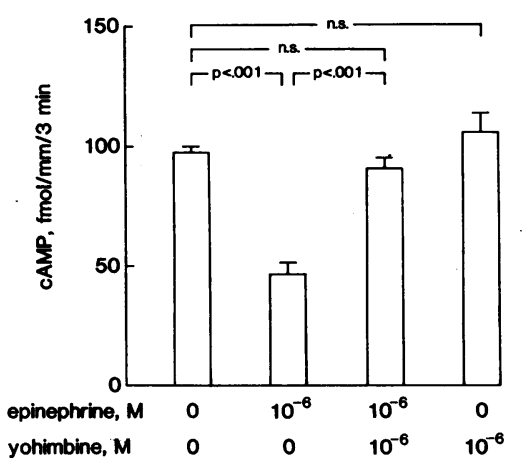

Figure 4. Effects of epinephrine and yohimbine on AVP-dependent CAMP production in MCT after preincubation without pertussis toxin for $3 \mathrm{~h}$. Epinephrine inhibited AVP-dependent CAMP production in MCT, which was abolished by a specific alpha ${ }_{2}$-adrenergic antagonist yohimbine. The means $\pm \mathrm{SE}$ of seven incubations of the results from a representative experiment are shown.

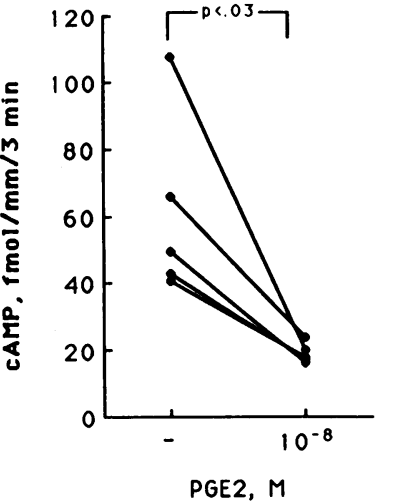

Figure 3. Effects of $10^{-6} \mathrm{M}$ epinephrine, $5 \mathrm{mM} \mathrm{Ca}^{2+}$, and $10^{-8} \mathrm{M} \mathrm{PGE}_{2}$ on AVP-dependent cAMP production in MAL after a 6-min preincubation without pertussis toxin. (See the legend of Fig. 2.) High ambient $\mathrm{Ca}^{2+}$ and $\mathrm{PGE}_{2}$ inhibited AVP-dependent CAMP production in MAL, but the suppression by epinephrine was trivial. not in MCT (14). As shown in Fig. 9, adenylate cyclase activity of permeabilized MAL and MCT was suppressed equally by micromolar concentrations of $\mathrm{Ca}^{2+}$; these results are similar to those reported previously (23). The distinct suppressive effect of high (5 mM) ambient $\mathrm{Ca}^{2+}$ on AVP-dependent cAMP production in intact MAL, but not in intact MCT, may be brought about through the interaction of extracellular $\mathrm{Ca}^{2+}$ with some machinery existing in the cell surface of MAL.

As shown in Fig. 10, PGE $_{2}$ suppressed AVP-dependent cAMP production in MAL at concentrations $>10^{-10} \mathrm{M}$ as shown previously in rat MAL (13). The suppression by $\mathrm{PGE}_{2}$ could be attenuated by pertussis toxin treatment. Thus, in MAL preincubated with pertussis toxin for $6 \mathrm{~h}$, significant suppression by $\mathrm{PGE}_{2}$ became evident at concentrations of PGE $_{2} 10^{-9} \mathrm{M}$, but not at $10^{-10} \mathrm{M}$, as shown in Fig. $10 a$. AVP-dependent cAMP production was significantly greater with pertussis toxin treatment than without at each concen-

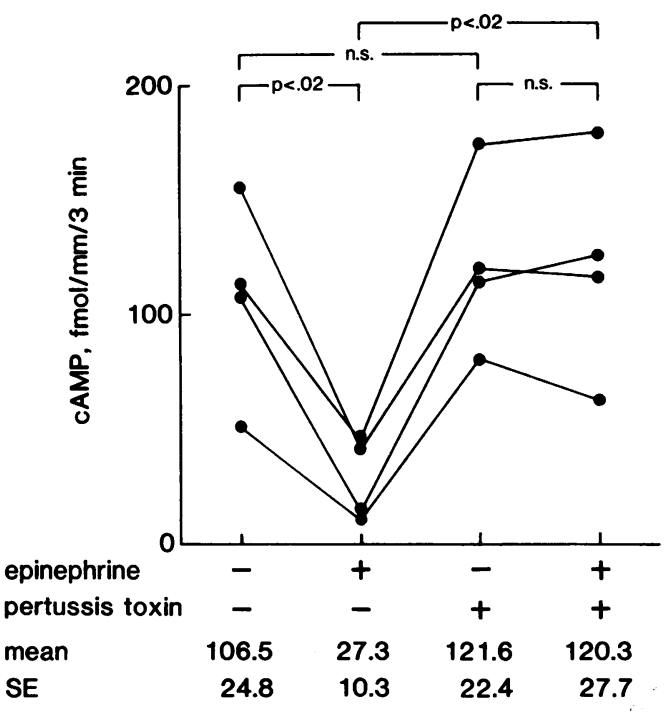

Figure 5. Effects of alpha ${ }_{2}$-adrenergic agonist epinephrine, $10^{-6} \mathrm{M}$, on AVP-dependent cAMP production in MCT aftér preincubation with or without pertussis toxin for $3 \mathrm{~h}$. Experiments were performed in the presence of $2 \times 10^{-6} \mathrm{M}$ propranolol. Each data point is the mean of values obtained from the MCT of one animal and the line connecting data points indicates the results obtained in the tubules of the same animal. Note that the inhibition by epinephrine was not observed after pertussis toxin treatment. Basal cAMP production without AVP in each condition was $<10 \mathrm{fmol} / \mathrm{mm}$. Means $\pm S E$ are given on the bottom. 


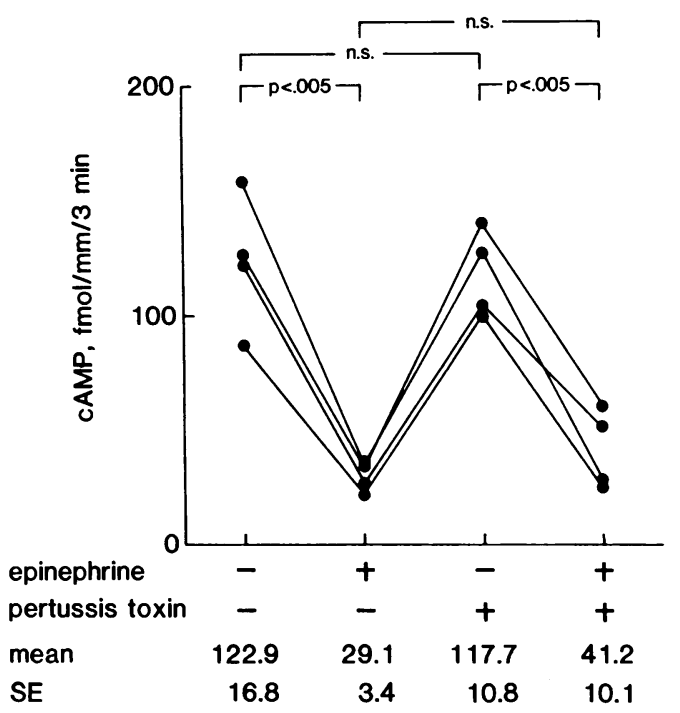

Figure 6. Effects of alpha ${ }_{2}$-adrenergic agonist epinephrine on AVPdependent CAMP production in MCT after preincubation with or without pertussis toxin for $1 \mathrm{~h}$. Note that pertussis toxin had little effect on AVP-dependent cAMP production and its inhibition by epinephrine. See the legend of Fig. 5.

tration of $\mathrm{PGE}_{2}$ (Fig. $10 \mathrm{~A}$ ). The magnitude of suppression by $\mathrm{PGE}_{2}$ with pertussis toxin treatment was significantly less than without at each $\mathrm{PGE}_{2}$ concentration $>10^{-10} \mathrm{M}$ (Fig. $10 \mathrm{~B}$ ). Thus, pertussis toxin treatment increased AVP-dependent cAMP production in each condition of $\mathrm{PGE}_{2}$ tested and attenuated the suppression by $\mathrm{PGE}_{2}$. As noted earlier, basal cAMP production was $<5 \mathrm{fmol} / \mathrm{mm}$ in each experimental condition. The effects of pertussis toxin in MAL, which are shown in Fig. $10, A$ and $B$, are due to its specific ADP-ribosylating action

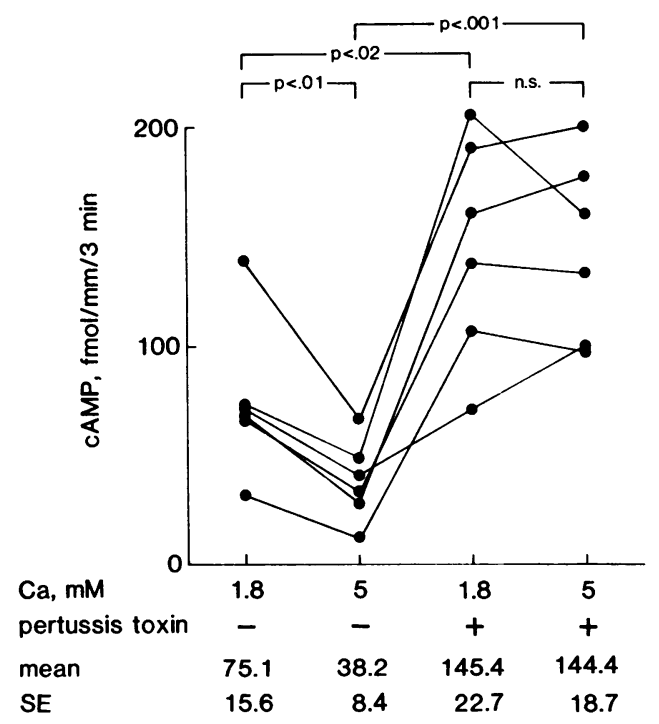

Figure 7. Effects of high ambient $\mathrm{Ca}^{2+}$ in the incubation medium on AVP-dependent cAMP production in MAL after preincubation with or without pertussis toxin for $6 \mathrm{~h}$. Note that the inhibition by $5 \mathrm{mM}$ $\mathrm{Ca}^{2+}$ was not observed after pertussis toxin treatment and that cAMP levels under $1.8 \mathrm{mM} \mathrm{Ca}^{2+}$ (controls) after pertussis toxin treatment were greater than those without pertussis toxin treatment. Basal cAMP production without AVP in each condition was $<5 \mathrm{fmol} /$ $\mathrm{mm}$. See the legend of Fig. 5 .

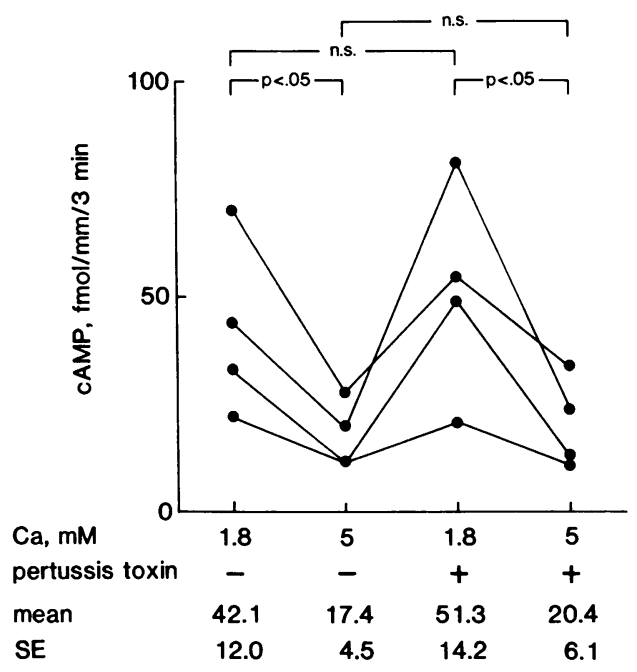

Figure 8. Effects of high ambient $\mathrm{Ca}^{2+}$ in the incubation medium on AVP-dependent cAMP production in MAL after preincubation with or without pertussis toxin for $1 \mathrm{~h}$. Note that pertussis toxin was without effect on AVP-dependent cAMP production and its inhibition by $5 \mathrm{mM}$ ambient $\mathrm{Ca}^{2+}$. See the legend of Fig. 7.

because they were not observed with MAL preincubated with pertussis toxin for $1 \mathrm{~h}$ (Fig. 11).

Data shown in the previous figures were summarized in Table I so that the effects of the length of preincubation time could be evaluated. The rate of AVP-dependent cAMP production without either pertussis toxin or any inhibitory agents, and the percentage of suppression by these inhibitory agents were comparable regardless of the length of preincubation time used in the present study. Thus, the tubules remained intact with the functional adenylate cyclase regulating system even after relatively long hours of preincubation.

\section{Discussion}

AVP increases water permeability in $\mathrm{MCT}$ and $\mathrm{NaCl}$ reabsorption in MAL through cAMP production (1-8). Alpha $2_{-}$ adrenergic agonists in MCT and high ambient $\mathrm{Ca}^{2+}$ and $\mathrm{PGE}_{2}$ in MAL suppress AVP-dependent cAMP production and thereby modulate urine concentration (10-15). We attempted to clarify the mechanisms underlying the suppression of AVPdependent cAMP production by these agents.

Our present study showed that both MCT and MAL possessed an $\sim 41-\mathrm{kD}$ protein that was specifically ADP-ribosylated by pertussis toxin. In the present study, preincubation with pertussis toxin of MCT for $3 \mathrm{~h}$ and of MAL for $6 \mathrm{~h}$ were needed for the full expression of the effect of pertussis toxin to ADP-ribosylate this $41-\mathrm{kD}$ protein. The lengths of the preincubation time needed for ADP-ribosylation by pertussis toxin are similar to those reported for other tissues $(17,18,21,24)$.

Our present data clearly show that epinephrine suppresses AVP-dependent cAMP production in MCT, which is blocked by yohimbine, and that this suppressive action of epinephrine is also abolished by pretreatment of MCT by pertussis toxin for $3 \mathrm{~h}$ but not for $1 \mathrm{~h}$. It has been well established by the data in other tissues that the inhibition of adenylate cyclase activity by alpha ${ }_{2}$-adrenergic stimulation is mediated through the activation of $G_{i}(16,17)$. These results indicate that the same mecha- 

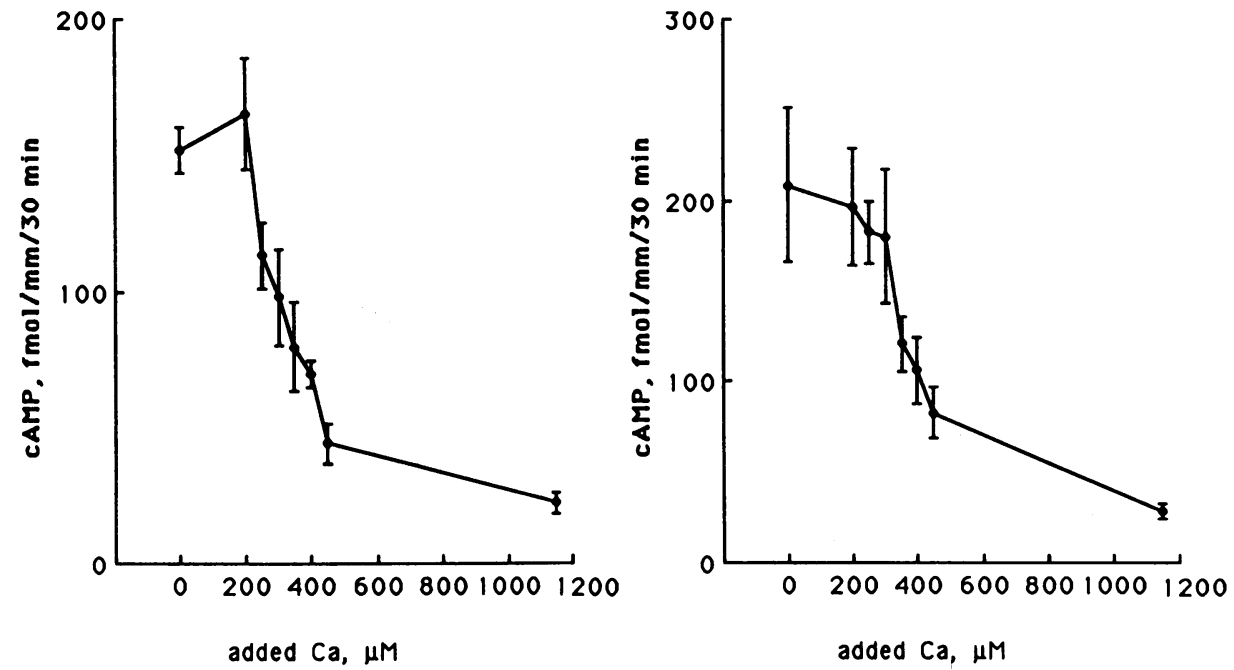

Figure 9. Effects of increasing concentration of $\mathrm{Ca}^{2+}$ on adenylate cyclase activity in permeabilized MAL (left) and MCT (right) in the presence of $250 \mu \mathrm{M}$ EGTA. Results of a representative experiment are shown. Note that micromolar range of $\mathrm{Ca}^{2+}$ equally inhibits adenylate cyclase activity in both nephron segments. Each data point is mean \pm SE of five to six incubations.

nism is operating in the action of alpha $a_{2}$-adrenergic stimulation in MCT to suppress AVP-dependent adenylate cyclase activation.

Our data also showed that the inhibition of AVP-dependent cAMP production by high ambient $\mathrm{Ca}^{2+}$ in MAL was abolished by pertussis toxin pretreatment of these tubular cells. The suppression of AVP-dependent cAMP production by $\mathrm{PGE}_{2}$ in MAL was also attenuated after pertussis toxin treatment. Moreover, in MAL, but not in MCT, AVP-dependent cAMP production in the control condition with AVP, but without inhibiting agents, became greater after treatment with pertussis toxin than that without pertussis toxin. The effects of pertussis toxin are due to its specific effect of being able to ADP-ribosylate a $41-\mathrm{kD}$ protein, presumably the alpha-subunit of $G_{i}(17,18)$ because the effects were not observed in MAL preincubated with pertussis toxin for only $1 \mathrm{~h}$, during which time ADP-ribosylation did not occur in our experimental condition. Based on the results of our present study, it is likely that the suppression of AVP-dependent cAMP produc-
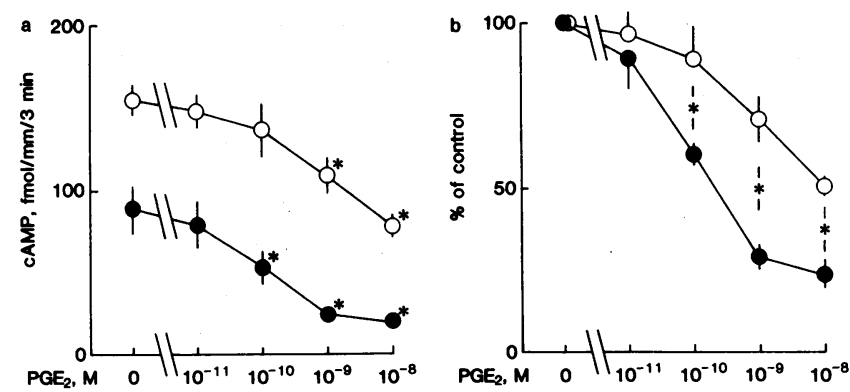

Figure 10. Effects of $\mathrm{PGE}_{2}$ on AVP-dependent cAMP production in MAL after preincubation with or without pertussis toxin for $6 \mathrm{~h}$. Results with pertussis toxin treatment are shown in open circles, and those without pertussis toxin treatment are shown in closed circles. (a) AVP-dependent cAMP production with and without $10^{-11}-10^{-8}$ $M P_{2}$. Basal cAMP production without AVP was $<5 \mathrm{fmol} / \mathrm{mm}$. Each data point represents mean \pm SE of 5 mice. Asterisks indicate a significant difference from respective control (without $\mathrm{PGE}_{2}$ ) values. $(B)$ Data in $A$ are expressed in percent of control to demonstrate the magnitude of suppression by $\mathrm{PGE}_{2}$. Asterisks indicate significant differences in the magnitude of suppression. tion by high ambient $\mathrm{Ca}^{2+}$ in MAL is mediated through the activation of $G_{i}$, and the suppression by $P E_{2}$ in MAL is also mediated, at least in part, through the activation of $G_{i}$. Since the suppression of AVP-dependent cAMP production by $\mathrm{PGE}_{2}$ in MAL was not completely prevented by pertussis toxin treatment in the presence of a high dose of $\mathrm{PGE}_{2}$, it is possible that a part of the suppression by $\mathrm{PGE}_{2}$ may be brought about through an interaction of $P E_{2}$ with $G_{s}$ as suggested by the results seen in collecting tubules (25). Moreover, our data on the reversal by pertussis toxin of the suppression of AVP-dependent cAMP production by $\mathrm{PGE}_{2}$ in MAL corroborate the observations that $P G E_{2}$ receptors may be linked to $G_{i}$ in the renal medulla (26), and that the suppression by PG of adenylate cyclase activity in adipocytes may be mediated through the activation of $G_{i}(18)$.

The mechanisms by which high ambient $\mathrm{Ca}^{2+}$ may activate $\mathrm{G}_{i}$ and thereby suppress AVP-dependent cAMP production in

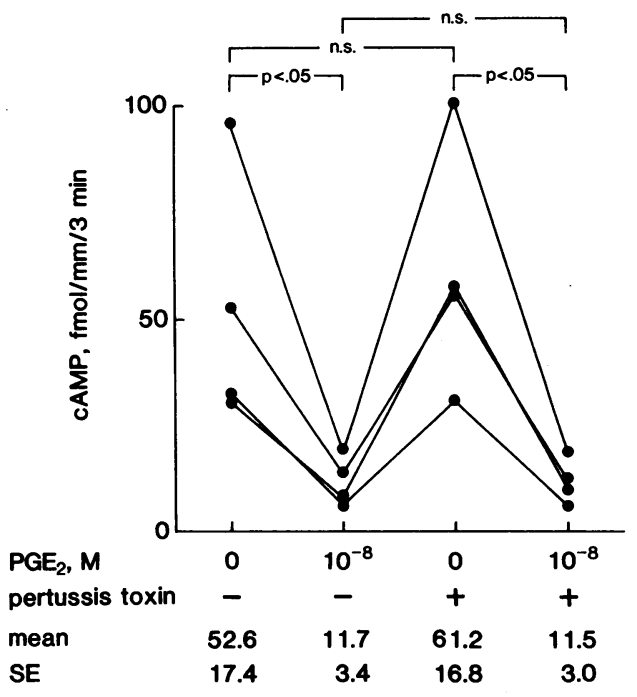

Figure 11. Effects of $10^{-8} \mathrm{M} \mathrm{PGE}_{2}$ on AVP-dependent cAMP production in MAL after preincubation with or without pertussis toxin for $1 \mathrm{~h}$. Note that $10^{-8} \mathrm{M} \mathrm{PGE}_{2}$ suppressed AVP-dependent cAMP production to a similar degree in both conditions and that AVP-dependent cAMP production without $\mathrm{PGE}_{2}$ in both conditions was comparable. See the legend of Fig. 5. 
Table I. Effects of Preincubation Time on AVP-dependent cAMP Production in MCT and MAL

\begin{tabular}{lccc}
\hline & \multicolumn{3}{c}{ Preincubation time } \\
\cline { 2 - 4 } & $6 \mathrm{~min}$ & $1 \mathrm{~h}$ & $3 \mathrm{~h}$ \\
\hline & & fmol/mm per $3 \mathrm{~min}$ \\
MCT & & & \\
Control & $149.2 \pm 17.1$ & $122.9 \pm 16.8$ & $106.5 \pm 24.8$ \\
With epinephrine & $47.7 \pm 7.4$ & $29.1 \pm 3.4$ & $27.3 \pm 10.3$ \\
\% Suppression & $67.5 \pm 5.6$ & $76.0 \pm 1.2$ & $75.4 \pm 6.4$ \\
No. of mice & 4 & 4 & 4 \\
& $6 \mathrm{~min}$ & $1 \mathrm{~h}$ & $6 \mathrm{~h}$ \\
\cline { 2 - 4 } MAL & \multicolumn{3}{c}{} \\
Control & $67.4 \pm 12.3$ & $42.1 \pm 12.0$ & $75.1 \pm 15.6$ \\
With 5 mM Ca & $31.7 \pm 4.9$ & $17.4 \pm 4.5$ & $38.2 \pm 8.4$ \\
\% Suppression & $50.6 \pm 7.7$ & $57.3 \pm 4.3$ & $50.2 \pm 4.7$ \\
No. of mice & 5 & 4 & 6 \\
Control & $64.3 \pm 12.4$ & $52.6 \pm 17.4$ & $88.5 \pm 14.6$ \\
With PGE & $19.1 \pm 1.5$ & $11.7 \pm 3.4$ & $19.3 \pm 2.6$ \\
\% suppression & $65.5 \pm 4.8$ & $77.3 \pm 2.0$ & $76.3 \pm 4.5$ \\
No. of mice & 5 & 4 & 5 \\
& & &
\end{tabular}

Cyclic AMP production ( $\mathrm{fmol} / \mathrm{mm}$ per $3 \mathrm{~min}$ ) and percent suppression (\%) by epinephrine $\left(10^{-6} \mathrm{M}\right)$ in MCT and by high ambient $\mathrm{Ca}^{2+}$ $(5 \mathrm{mM})$ and $\mathrm{PGE}_{2}\left(10^{-8} \mathrm{M}\right)$ are shown. Note that cAMP production in the absence of any inhibitors (controls) and percent suppression by the inhibitors were comparable regardless of the length of preincubation time. Isolated tubules were preincubated without pertussis toxin. Values are the mean $\pm \mathrm{SE}$ and the numbers of mice for each experiment are given at the bottom of each set of experiment.

MAL is not clear. As might be expected from looking at the available data (23), adenylate cyclase activation by AVP in permeabilized tubular cell preparations were inhibited equally in both MCT and MAL by a micromolar concentration of $\mathrm{Ca}^{2+}$ (Fig. 9). By contrast, inhibition by high ambient $\mathrm{Ca}^{2+}$ of cAMP production in intact cell preparations was seen only in MAL and not in MCT. These results suggest that high extracellular $\mathrm{Ca}^{2+}$ suppresses AVP-dependent cAMP production in MAL, probably through the interaction of extracellular $\mathrm{Ca}^{2+}$ with some machinery existing on the plasma membranes of this nephron segment. It is also indicated that this mechanism is sensitive to pertussis toxin. Thus, it may be possible that MAL possesses $\mathrm{aCa}^{2+}$ receptor on the cell surface that is linked to $G_{i}$ and is similar to those proposed in parathyroid cells (27, 28 ). These possibilities are only speculative and need further study.

It is of note that AVP-dependent cAMP production in MAL, but not in MCT, became greater after $6 \mathrm{~h}$ of preincubation with pertussis toxin. These data may suggest the possibility that $G_{i}$ is relatively dominant over $G_{s}$ in $M A L$, which may have some functional significance in the hormonal regulation of MAL (29). Alternately, it is possible that AVP-sensitive cAMP production without $\mathrm{PGE}_{2}$ or high ambient $\mathrm{Ca}^{2+}$ was partially suppressed via $\mathrm{G}_{i}$ activation by the $1.8 \mathrm{mM} \mathrm{Ca}^{2+}$ present in the control incubation medium, and that pertussis toxin prevented this $G_{i}$ activation under control conditions.

Despite relatively long hours of preincubation, we believe that the tubules remained intact with the functional adenylate cyclase regulating system for the following reasons. First, as shown in the Results, the rates of AVP-dependent cAMP production without pertussis toxin or any inhibitory agents are comparable in both nephron segments preincubated for varied lengths of time (Table I). This indicates that tubules possess an intact adenylate cyclase-stimulating system even after long hours (3-6 h) of preincubation. Second, the magnitudes of suppression by epinephrine in MCT and by high ambient $\mathrm{Ca}^{2+}$ and $\mathrm{PGE}_{2}$ in MAL without pertussis toxin are also comparable regardless of the length of preincubation time (Table I), indicating that adenylate cyclase-inhibiting systems sensitive to these agents also remain intact during the preincubation condition employed in the present study.

In summary, our present study showed that ADP-ribosylation of a $41-\mathrm{kD}$ protein by pertussis toxin abolished or attenuated the inhibition of AVP-dependent cAMP production by alpha ${ }_{2}$-adrenergic stimulation in MCT and by high ambient $\mathrm{Ca}^{2+}$ and $\mathrm{PGE}_{2}$ in MAL. Moreover, pertussis toxin pretreatment resulted in an increase in AVP-dependent cAMP production in the absence of these inhibitory agents in MAL but not in MCT. These data suggest that the inhibition of AVPdependent cAMP production by alpha ${ }_{2}$-adrenergic stimulation in MCT, and by high ambient $\mathrm{Ca}^{2+}$ and $\mathrm{PGE}_{2}$ in MAL are, at least in part, mediated through the activation of $\mathrm{G}_{i}$.

\section{Acknowledgments}

We thank Dr. Etsuro Ogata and Dr. Itaru Kojima for invaluable suggestions, Ms. Kazuko Sekizaki for technical assistance, and Ms. Satomi Namioka for secretarial assistance. This study was partly supported by the grants from the Ministry of Education and the Ministry of Health and Welfare of Japan.

\section{References}

1. Grantham, J. J., and J. Orloff. 1968. Effect of prostaglandin $E_{1}$ on the permeability response of the isolated collecting tubule to vasopressin, adenosine 3',5'-monophosphate, and theophylline. J. Clin. Invest. 47:1154-1164.

2. Grantham, J. J., and M. B. Burg. 1966. Effect of vasopressin and cyclic AMP on permeability of isolated collecting tubules. Am. J. Physiol. 211:255-259.

3. Hall, D. A., and D. M. Varney. 1980. Effect of vasopressin on electrical potential difference and chloride transport in mouse medullary thick ascending limb of Henle's loop. J. Clin. Invest. 66:792-802.

4. Hebert, S. C., R. M. Culpepper, and T. E. Andreoli. 1981. NaCl transport in mouse medullary thick ascending limbs. I. Functional nephron heterogeneity and $\mathrm{ADH}$-stimulated $\mathrm{NaCl}$ cotransport. Am. J. Physiol. 241:F412-F431.

5. Jamison, R. L., and R. H. Maffly. 1976. The urinary concentrating mechanism. N. Engl. J. Med. 295:1059-1067.

6. Kim, J. K., S. N. Summer, A. E. Erickson, and R. W. Schrier. 1986. Role of arginine vasopressin in medullary thick ascending limb on maximal urinary concentration. Am. J. Physiol. 251:F266-F270.

7. Morel, F. 1981. Sites of hormone action in the mammalian nephron. Am. J. Physiol. 240:F159-F164.

8. Sasaki, S., and M. Imai. 1980. Effects of vasopressin on water and $\mathrm{NaCl}$ transport across the in vitro perfused medullary thick ascending limb of Henle's loop of mouse, rat, and rabbit kidneys. Pfluegers Arch. Eur. J. Physiol. 383:215-221.

9. Rodbell, M. 1980. The role of hormone receptors and GTP-regulatory proteins in membrane transduction. Nature (Lond.). 284:17-21.

10. Krothapalli, R. K., and W. N. Suki. 1984. Functional characterization of the alpha adrenergic receptor modulating the hydroos- 
motic effect of vasopressin on the rabbit cortical collecting tubule. $J$. Clin. Invest. 73:740-749.

11. Chabardes, D., M. Montegut, M. Imbert-Teboul, and F. Morel. 1984. Inhibition of $\alpha_{2}$-adrenergic agonists on AVP-induced cAMP accumulation in isolated collecting tubule of the rat kidney. Mol. Cell. Endocrinol. 37:263-275.

12. Umemura, S., D. Marver, D. D. Smyth, and W. A. Pettinger. 1985. $\alpha_{2}$-adrenoreceptors and cellular cAMP levels in single nephron segments from the rat. Am. J. Physiol. 249:F28-F33.

13. Torikai, S., and K. Kurokawa. 1983. Effect of $\mathrm{PGE}_{2}$ on vasopressin-dependent cell cAMP in isolated single nephron segments. Am. J. Physiol. 245:F58-F66.

14. Takaichi, K., S. Uchida, and K. Kurokawa. 1986. High $\mathrm{Ca}^{2+}$ inhibits AVP-dependent cAMP production in thick ascending limbs of Henle. Am. J. Physiol. 250:F770-F776.

15. Culpepper, R. M., and T. E. Andreoli. 1983. Interactions among prostaglandin $\mathrm{E}_{2}$, antidiuretic hormone, and cyclic adenosine monophosphate in modulating $\mathrm{Cl}^{-}$absorption in single mouse medullary thick ascending limbs of Henle. J. Clin. Invest. 71:1588-1601.

16. Michel, T., and R. J. Lefkowitz. 1982. Hormonal inhibition of adenylate cyclase. $\alpha_{2}$ adrenoreceptors promote release of $\left[{ }^{3} \mathrm{H}\right] \mathrm{guanyly}$ limidodiphosphate from platelet membranes. J. Biol. Chem. 257:13557-13563.

17. Kurose, H., T. Katada, T. Amano, and M. Ui. 1983. Specific uncoupling by islet-activating protein, pertussis toxin, of negative signal transduction via $\alpha$-adrenergic, cholinergic, and opiate receptors in neuroblastoma $\times$ glioma hybrid cells. J. Biol. Chem. 258:4870-4875.

18. Murayama, T., and M. Ui. 1983. Loss of the inhibitory function of the guanine nucleotide regulatory component of adenylate cyclase due to its ADP ribosylation by islet-activating protein, pertussis toxin, in adipocyte membranes. J. Biol. Chem. 258:3319-3326.

19. Torikai, S., M.-S. Wang, K. L. Klein, and K. Kurokawa. 1981. Adenylate cyclase and cell cyclic AMP of rat cortical thick ascending limb of Henle. Kidney Int. 20:649-654.
20. Takaichi, K., and K. Kurokawa. 1986. High $\mathrm{Ca}^{2+}$ inhibits peptide hormone-dependent cAMP production specifically in thick ascending limbs of Henle. Miner. Electrolyte Metab. 12:342-346.

21. Okajima, F., T. Katada, and M. Ui. 1985. Coupling of the guanine nucleotide regulatory protein to chemotactic peptide receptors in neutrophil membranes and its uncoupling by islet-activating protein, pertussis toxin. A possible role of the toxin substrate in $\mathrm{Ca}^{2+}$. mobilizing receptor-mediated signal transduction. J. Biol. Chem. 260:6761-6768.

22. Kurokawa, K., R. M. Friedler, and S. G. Massry. 1975. Renal action of cholera toxin. II. Effects on adenylate cyclase-cyclic AMP system. Kidney Int. 7:137-144.

23. Marumo, F., and I. S. Edelman. 1971. Effects of $\mathrm{Ca}^{++}$and prostaglandin $E_{1}$ on vasopressin activation of renal adenyl cyclase. $J$. Clin. Invest. 50:1613-1620.

24. Arend, L. J., W. K. Sonnenburg, W. L. Smith, and W. S. Spielman. 1987. $A_{1}$ and $A_{2}$ adenosine receptors in rabbit cortical collecting tubule cells. Modulation of hormone-stimulated cAMP. $J$. Clin. Invest. 79:710-714.

25. Nadler, S. P., S. C. Hebert, and B. M. Brenner. 1986. PGE , forskolin, and cholera toxin interactions in rabbit cortical collecting tubule. Am. J. Physiol. 250:F127-F135.

26. Watanabe, T., K. Umegaki, and W. L. Smith. 1986. Association of a solubilized prostaglandin $E_{2}$ receptor from renal medulla with a pertussis toxin-reactive guanine nucleotide regulatory protein. $J$. Biol. Chem. 261:13430-13439.

27. Nemeth, F. N., and A. Scarpa. 1987. Rapid mobilization of cellular $\mathrm{Ca}^{2+}$ in bovine parathyroid cells evoked by extracellular divalent cations. Evidence for a cell surface calcium receptor. J. Biol. Chem. 262:5188-5196.

28. Wolf, F., and A. Scarpa. 1987. Calcium binding by parathyroid cell plasma membranes. Cell Calcium. 8:171-183.

29. Cerione, R. A., C. Staniszewski, M. G. Caron, R. J. Lefkowitz, J. Codina, and L. Birnbaumer. 1985. A role for $N_{i}$ in the hormonal stimulation of adenylate cyclase. Nature (Lond.). 318:293-295. 